Keywords:

Ion Exchange

High Level Waste

Cesium-137

Radiation Monitors

Retention Time: Permanent

\title{
Small Column Ion Exchange Monitor System Final Report
}

\section{V.R. Casella, R. A. Sigg, F. S. Moore, T.N. Riley and S.R. Salaymeh}

\section{Publication Date: September 30, 2004}

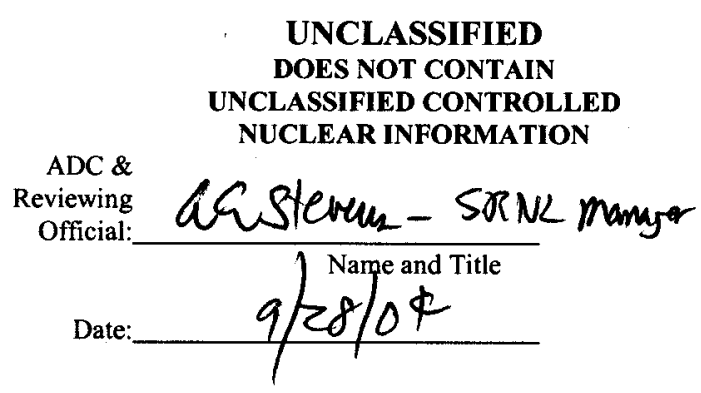

Westinghouse Savannah River Company

Savannah River Site

Aiken, SC 29808

Prepared for the U.S. Department of Energy Under

Contract Number DE-AC09-96SR18500

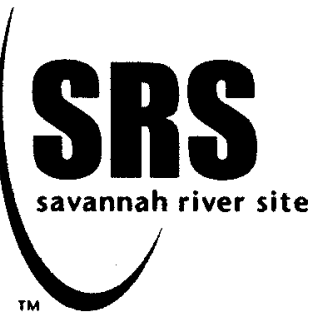


This document was prepared in conjunction with work accomplished under Contract No. DE-AC09-96SR18500 with the U. S. Department of Energy.

\section{DISCLAIMER}

This report was prepared as an account of work sponsored by an agency of the United States Government. Neither the United States Government nor any agency thereof, nor any of their employees, makes any warranty, express or implied, or assumes any legal liability or responsibility for the accuracy, completeness, or usefulness of any information, apparatus, product or process disclosed, or represents that its use would not infringe privately owned rights. Reference herein to any specific commercial product, process or service by trade name, trademark, manufacturer, or otherwise does not necessarily constitute or imply its endorsement, recommendation, or favoring by the United States Government or any agency thereof. The views and opinions of authors expressed herein do not necessarily state or reflect those of the United States Government or any agency thereof.

This report has been reproduced directly from the best available copy.

Available for sale to the public, in paper, from: U.S. Department of Commerce, National Technical Information Service, 5285 Port Royal Road, Springfield, VA 22161, phone: (800) 553-6847, fax: (703) 605-6900

email: orders@ntis.fedworld.gov

online ordering: http://www.ntis.gov/help/index.asp

Available electronically at http://www.osti.gov/bridge

Available for a processing fee to U.S. Department of Energy and its contractors, in paper, from: U.S. Department of Energy, Office of Scientific and Technical Information, P.O. Box 62, Oak Ridge, TN 37831-0062,

phone: (865)576-8401,

fax: (865)576-5728

email: $\underline{\text { reports@ adonis.osti.gov }}$ 
This page was intentionally left blank 
WSRC-RP-2004-00615

Revision 0

- iv -

TABLE OF CONTENTS

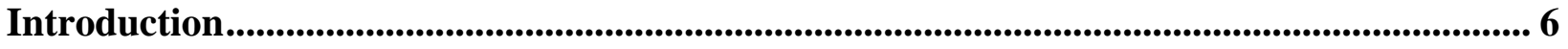

System Description .......................................................................................................... 6

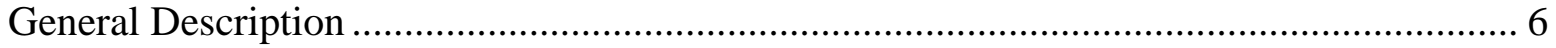

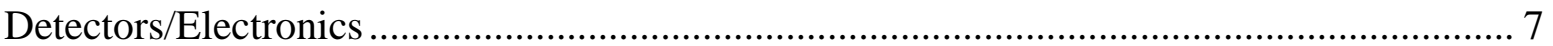

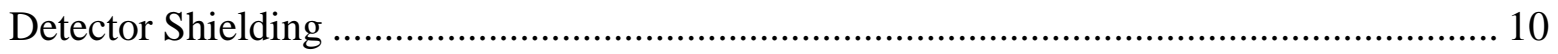

Detector Calibration.................................................................................................. 14

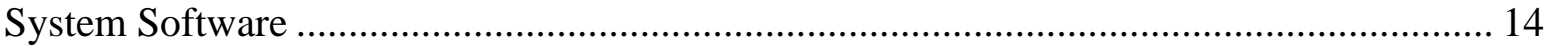

References...................................................................................................................................... 15

Appendix 1-Software User's Manual .............................................................................. 16

Appendix 2-Software Test Plan ........................................................................................................... 28 
Table 1. Simplfied process monitoring schematic ..................................................................................... 7

Table 2. Ba-137m decay and Ba-137m ingrowth for $\mathrm{Cs}-137$............................................................. 7

Table 3. Process monitoring electronics diagram ............................................................................ 9

Table 4. ORNL Shield Design .............................................................................................. 11

Table 5. Side view of shielding model................................................................................. 11

Table 6. Front view line drawing of the shielded model................................................................ 12

Table 7. View of the shielding model including the process pipe running beside the detector and

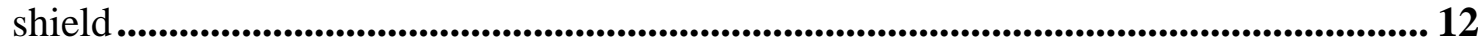

Table 8. Line drawing showing gamma-ray tracks from the process pipe to the NaI detector ... 13

Table 9. Standard 1-in diameter x 2-in long schedule 40 pipe containing 0.5 mCi Cs-137 ........ 14

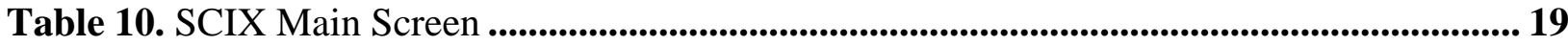

Table 11. Configuration Utility Password Screen.......................................................................... 20

Table 12. Detector Configuration Screen.................................................................................... 21

Table 13. Routine Ion Exchange Data - GM Graph............................................................................ 22

Table 14. PMT Data - NaI Detector Graph................................................................................ 23

Table 15. Ba-137m Decay / Ba-137m from Cs-137 Ingrowth Graph............................................... 24

Table 16. Spent Resin Operation - GM Graph............................................................................ 25 


\section{INTRODUCTION}

A Small Column Ion Exchange (SCIX) system has been designed by the Oak Ridge and Savannah River National Laboratories (ORNL and SRNL) as a potential way to reduce Cs137 concentrations in high-level radioactive waste at the Savannah River Site. SRNL was asked to develop gamma-ray monitors at six locations within the SCIX system. Gamma-ray monitors are required to verify the proper operation of the ion exchange system, detect cesium breakthrough, and confirm presence of cesium before and after used resin is transferred to a grinder module.

The only observable gamma ray in the decay of Cs-137 is from its short-lived Ba-137m daughter. Chemical processes, such as the SCIX, may disrupt the secular equilibrium between this parent-daughter pair; meaning that measurement of Ba-137m will not necessarily yield information about Cs-137 content. While this is a complicating factor that can not be ignored, it is controllable by either: allowing sufficient time for equilibrium to be reestablished (about 20 minutes), or by making multiple measurements with sufficient statistical precision to determine the extent of disequilibrium. The present work provides a means of measuring the Cs- 137 and $\mathrm{Ba}-137 \mathrm{~m}$ by taking multiple measurements in a process isolation loop that contains the process solution of interest.

This work is supported by the DOE Office of Accelerated Cleanup through award to ORNL and SRNL on their funded project entitled "Small Column Ion Exchange System Utilizing Silicotitinate for Cesium Removal from Low Curie Salt Waste (Phase 2)."

\section{SYSTEM DESCRIPTION}

\section{General Description}

Gamma-ray monitors have been provided at six SCIX locations for the purposes of measuring Cs-137 breakthrough and for monitoring normal transfers of radioactive materials as shown in Figure 1. Two sodium iodide breakthrough monitors, one Geiger-Mueller (GM) breakthrough monitor, and three Geiger-Mueller transfer monitors are shown. $\mathrm{GM}_{2}$ is a breakthrough monitor for the ion exchange column, while $\mathrm{GM}_{1}$ monitors the solution radioactivity before the column, and $\mathrm{GM}_{3}$ and $\mathrm{GM}_{4}$ monitor the spent-column slurry when it is being transferred through the grinder.

Two thallium-doped sodium iodide, $\mathrm{NaI}(\mathrm{Tl})$, gamma-ray spectrometers provide redundant, quantitative Cs-137 concentration data. Each uses a flow diversion and isolation loop. Once the sample is isolated, data acquisition protocols provide a rapid succession of short duration counts, and these are analyzed to determine the current Cs-137 / Ba-137m equilibrium. In the event that one system fails, the other is able to provide equivalent data. While reaching equilibrium is not a necessary condition, final analytical results will be determined more precisely if measurements are made at that time. 


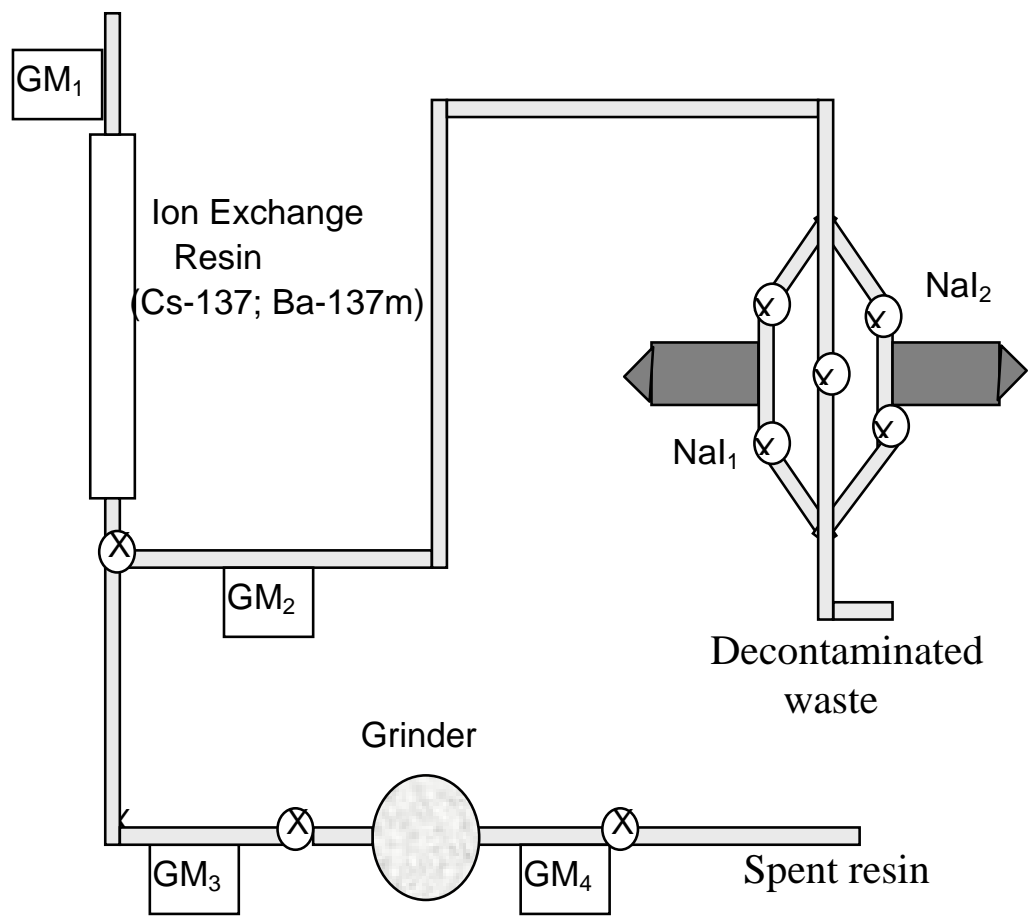

Figure 1. Simplified process monitoring schematic.

\section{Detectors/Electronics}

The two NaI detectors are used to track the buildup or decay of Cs-137. Samples of the ion exchange column eluant are taken into an isolated pipe and counted repeatedly. Cs-137 beta decays to Ba-137m which decays to the ground state with the emission of a $661.7 \mathrm{KeV}$ gamma ray. The rate of buildup or decay of the $661.7 \mathrm{KeV}$ gamma ray from the Ba- $137 \mathrm{~m}$ is used to determine the condition of the ion exchange medium.

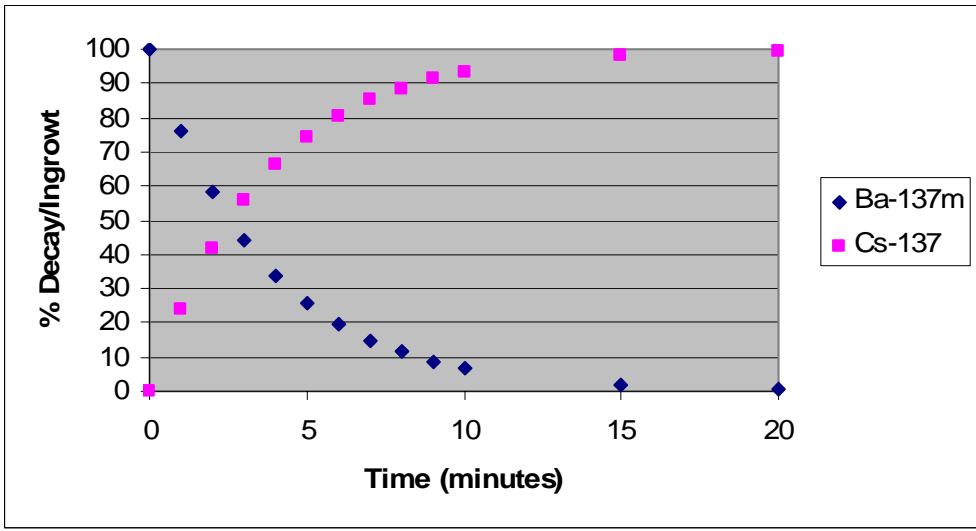

Figure 2. Ba-137m decay (blue points ) and Ba-137m ingrowth for Cs-137 (pink points). 
As shown in Figure 2, if there is only Ba-137m breakthrough, it will decay in a manner represented by the blue points in this graph. However, if there is only Cs-137 breakthrough, the Ba-137m ingrowth is shown by the pink points.

The two detectors are used, while one is analyzing a sample the other is off line. NaI detectors are used for this purpose because the output of the detector depends on the energy of the incident gamma ray. In this way the counts from the $661.7 \mathrm{KeV}$ gamma ray may be separated from other gamma-ray pulses by using a multichannel analyzer.

As stated above, four Geiger-Mueller (GM) detectors are also included. $\mathrm{GM}_{1}$ is placed adjacent to the input line into the ion exchange column. Its purpose is to indicate that material is flowing into the column. When material containing Cs-137 is flowing into the column or isolated above the column, it will read high. The reading will decrease if the material in the piping before the column does not contain as much Cs-137. GM 2 is placed near the output of the column, before the sampling line to the NaI detector. It will read high if an unexpected catastrophic failure in the column sends untreated Cs-137 bearing liquids out of the column. The $\mathrm{GM}_{3}$ detector is placed adjacent to the inlet to the grinder. It will read only high if the contents of the column are being dumped to the grinder. The $\mathrm{GM}_{4}$ detector is placed adjacent to the outlet of the grinder. It will read high only when material is flowing from the grinder into the storage tank.

A diagram of the process monitoring electronics is shown in Figure 3. The GM detectors are connected to Aware Electronics PMI-30 modules that convert the non-standard pulses into standard pulses. These PMI-30 modules have been mounted in nuclear instrument modules (NIMs) for convenience and to provide power to the PMI-30. Two PMI-30s are in each of two NIMs. The outputs of the GM detectors go to the PMI-30s in the NIM. Outputs from the PMI-30s go from the NIMs to a National Instruments BNC-2121 that converts the pulses to transistor-transistor logic (TTL) pulses. The BNC-2121 is interfaced to a National Instruments 6602 card mounted in a slot in the desktop PC. The control software obtains count rates from the GM detectors via the BNC-2121 and the 6602.

The signal processing electronics are primarily NIM standard modules, mounted in a NIM bin. This was chosen because the NIM standard is internationally known. The NIM bin provides the power for each individual module, through standard pin location on the bin backplane. The standard prescribes the sizes of individual modules, as well as standard input and output signal voltages and pulse widths. Modules (i.e., amplifiers, power supplies, scalars, etc.) from many vendors can be used in the same bin with confidence that signals will be processed properly. Spares are also simplified since all manufacturers adhere to this standard, and replacement of, for example, an amplifier by one from a different vendor can be done with confidence the system will perform as it did originally. 


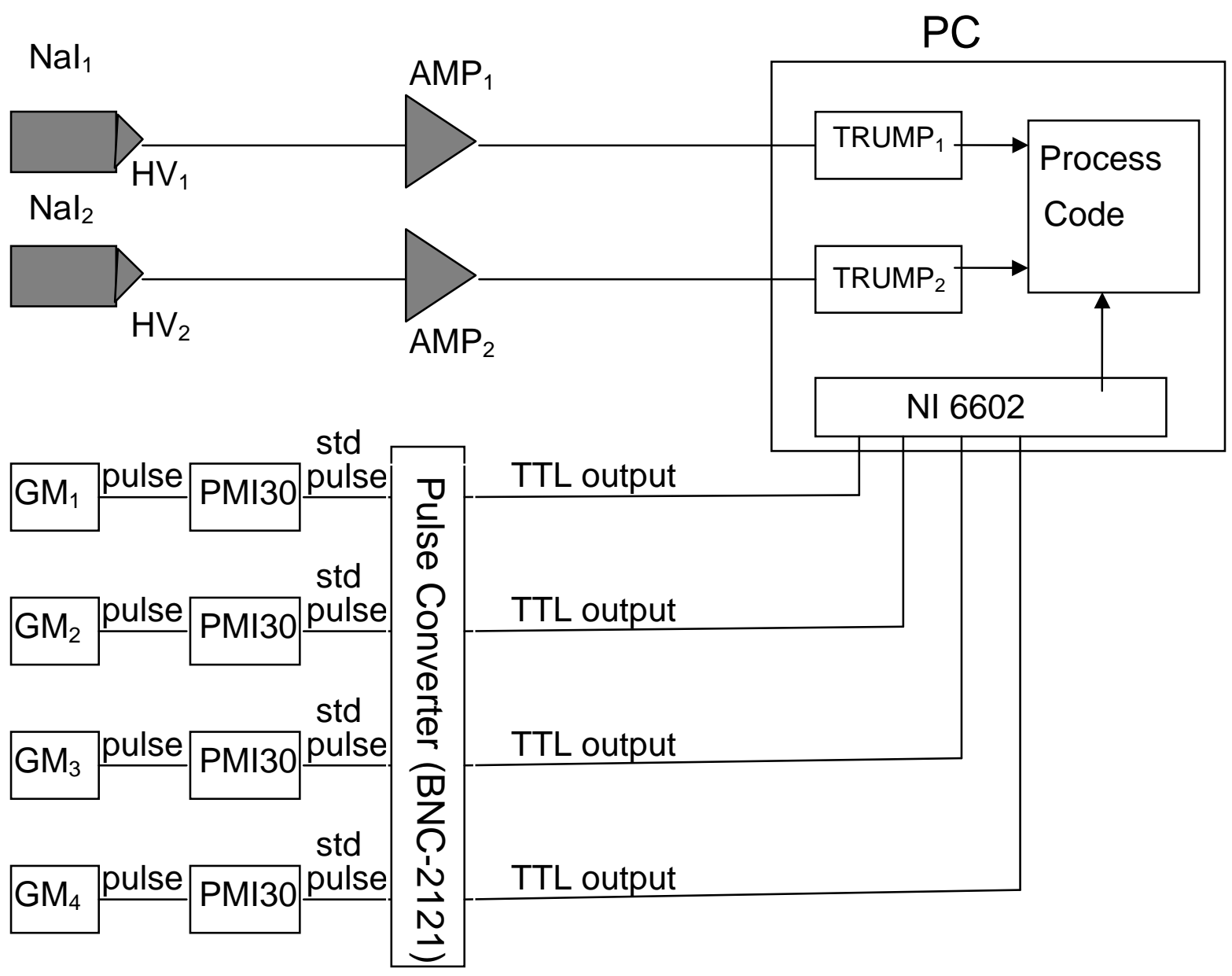

Figure 3. Process monitoring electronics diagram.

To reduce costs in the prototype system, "on hand" modules were used. The two amplifiers, one for each NaI detector, were Canberra 2015A, AMP/TSCAs. One high voltage power supply was an ORTEC 478A while the other was a Canberra 3005. An on hand NIM bin was used. For the production system, the following modules are acceptable (equivalent modules can also be used):

NIM Bin/power supply (1)

Amplifier (2)

High Voltage Power Supply (2)

Multichannel Analyzer card (2)

$\mathrm{NaI}$ detector (2)

GM detector (4)

PMI-30, mounted in NIM module (4)

National Instruments BNC-2121*

National Instruments 6602*

Desktop PC
ORTEC 4001A/4002A

CANBERRA 2015A*

ORTEC 478

ORTEC TRUMP-PCI*

Scionix 25B25/2-E2-X (hermetically sealed)* Ludlum 133-6, waterproof with $100 \mathrm{ft}$ cable* Aware Electronics PMI-30*

* signifies that items are on hand for the project. 
Each Scionix NaI detector obtains its high voltage from a separate high voltage power supply. Preamp power for each Scionix NaI detector is taken from the 9 pin "D" connector on the back of the amplifier module. The signal from the Scionix NaI detector is supplied to the "amp in" connector on the same amplifier module. RG-62U coaxial cable should be used to connect the output connector of the amplifier module to the input connector of the multichannel analyzer on the back of the PC. RG 62U coaxial cable is also used to connect the PMI-30 outputs to the BNC-2121.

The multichannel analyzer (MCA) is a TRUMP-PCI ${ }^{\mathrm{TM}}$ card installed in a spare slot in the control desktop PC. The Trump card was selected for this application because it is available as a card using a PCI slot in the PC. Other available MCA cards use the older ISA bus, and could be used if a compatible PC is used. Current desktop PCs have PCI slots, and few if any of the older ISA bus slots. Hence, use of the TRUMP-PCI card means that any current desktop PC can be used as the control computer. There are two TRUMP cards in the PC, one for each $\mathrm{NaI}$ detector.

After amplification and pulse shaping, all the detector signals are supplied to boards installed in a desktop PC, where software analyzes the signals and displays the resulting data on menu driven screens. The two ORTEC TRUMP cards receive the input data from the two Scionix NaI detectors. The TRUMP cards are full featured multichannel analyzer (MCA) cards which permit the PC to function as an MCA. In the initial setup of the system, each TRUMP card is run as a standalone MCA. A region of interest (ROI) is established around the Cs137 photopeak so that the total number of counts in this photopeak may be obtained by the operating software whenever required. In this way the total number of counts in the Cs-137 photopeak is acquired by the operating software for analysis. After they have been set up, they run in the background during operation of the system.

\section{Detector Shielding}

The Oak Ridge shielding design for the NaI detectors is shown in Figure 4. It is 8.625 inches in outside diameter including the steel outer case. Monte Carlo analyses were made to evaluate the response of the $\mathrm{NaI}$ detector to $0.1 \mathrm{Ci} / \mathrm{gallon} \mathrm{Cs}-137$ in the sampling pipe, and in a process pipe running behind the detector/shield assembly. 


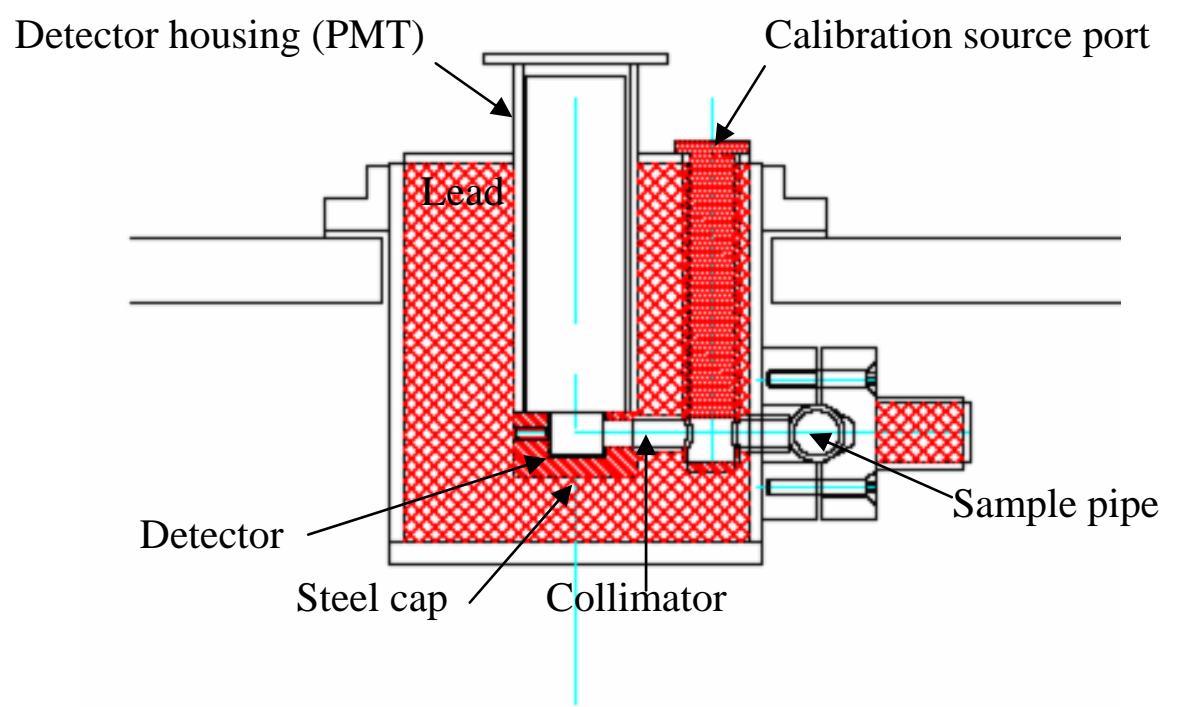

Figure 4. ORNL shield design.

Monte Carlo analyses track individual gamma rays from their birth in the source (i.e., here the sample pipe adjacent to the collimator) to their eventual capture or escape from the system. When a large enough number of particles have been traced, one can calculate the effectiveness of the shield/detector system. Monte Carlo N-Particle (MCNP) is one of the most used programs for Monte Carlo analyses. It is internationally applied to a variety of shielding problems. Figure 5 is a side view of the model, showing the important features modeled in MCNP; i.e., the detector, steel cap over it, large lead shield, collimator and the sample pipe.

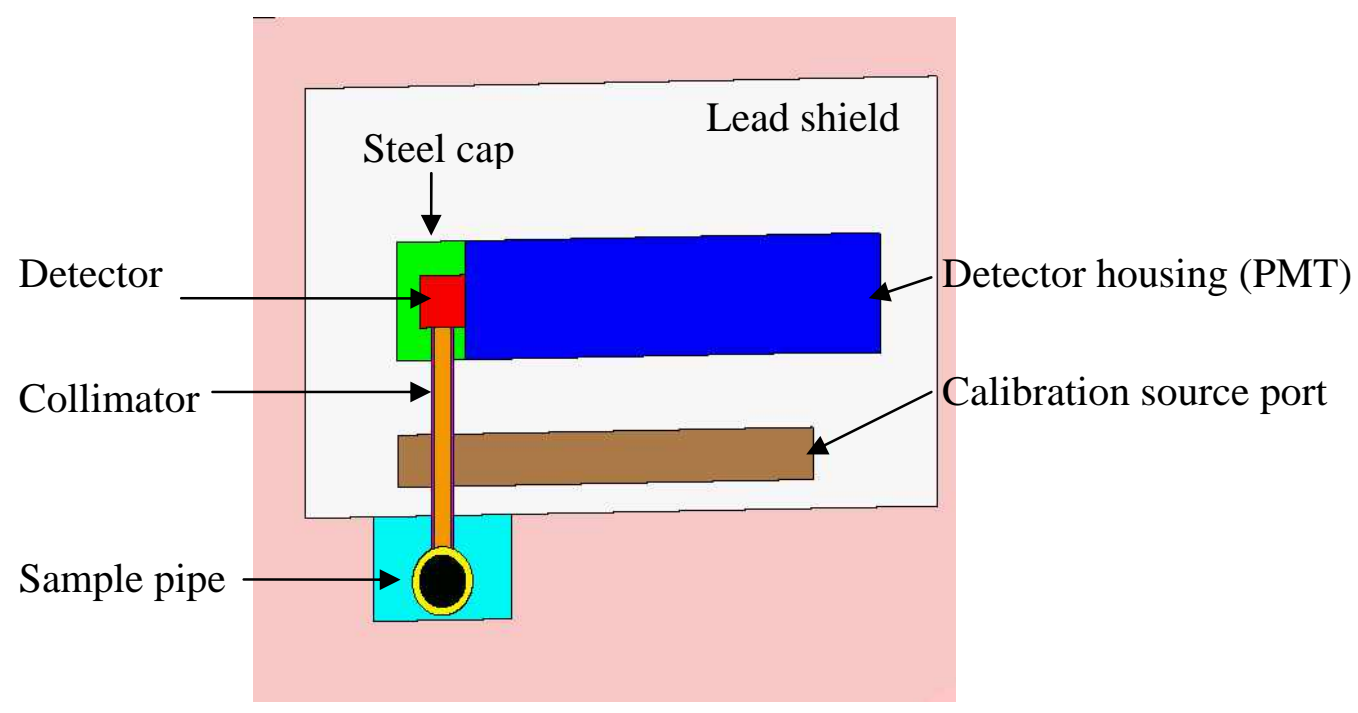

Figure 5. Side view of shielding model.

Figure 6 shows a front view line drawing of the model. In it are tracked gamma rays from the source pipe reaching the $\mathrm{NaI}$ detector. It can be seen that the collimator is doing an excellent 
job of limiting the gamma rays seen by the NaI detector to those coming from that part of the source pipe directly below the collimator. Even though gamma rays are emitted along the entire length of the pipe, out of 1.5 million tracked, none from the ends of the pipe reached the detector.

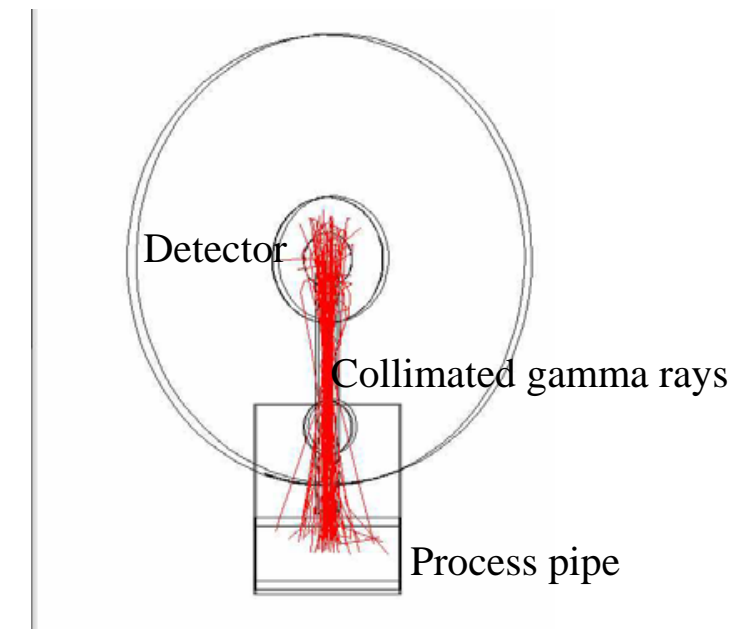

Figure 6. Front view line drawing of the shielding model.

Figure 7 is a view of the model including the process pipe running beside the detector and shield. This was used in MCNP to determine the effectiveness of the shield in limiting the number of gamma ray photons from the process pipe reaching the detector.

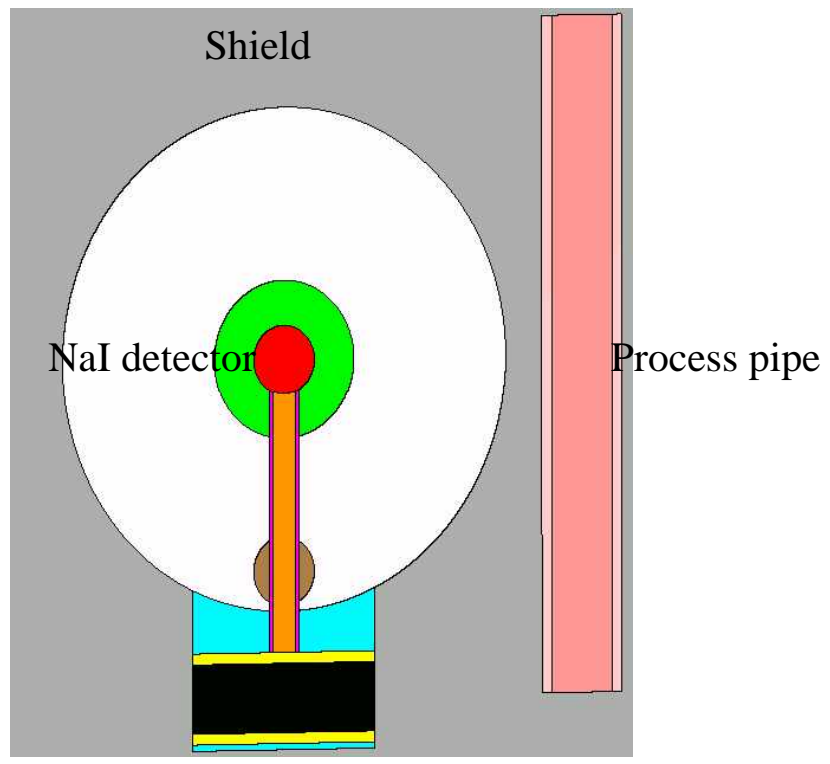

Figure 7. View of the shielding model including the process pipe running beside the detector and shield.

Figure 8 is a line drawing showing gamma ray tracks from the process pipe to the NaI detector. This is the result of following 100,000,000 gamma rays emitted from the entire 
length of the process pipe. It is apparent that only that portion of the process pipe adjacent to the shield contributes to the background gamma rays at the NaI detector.

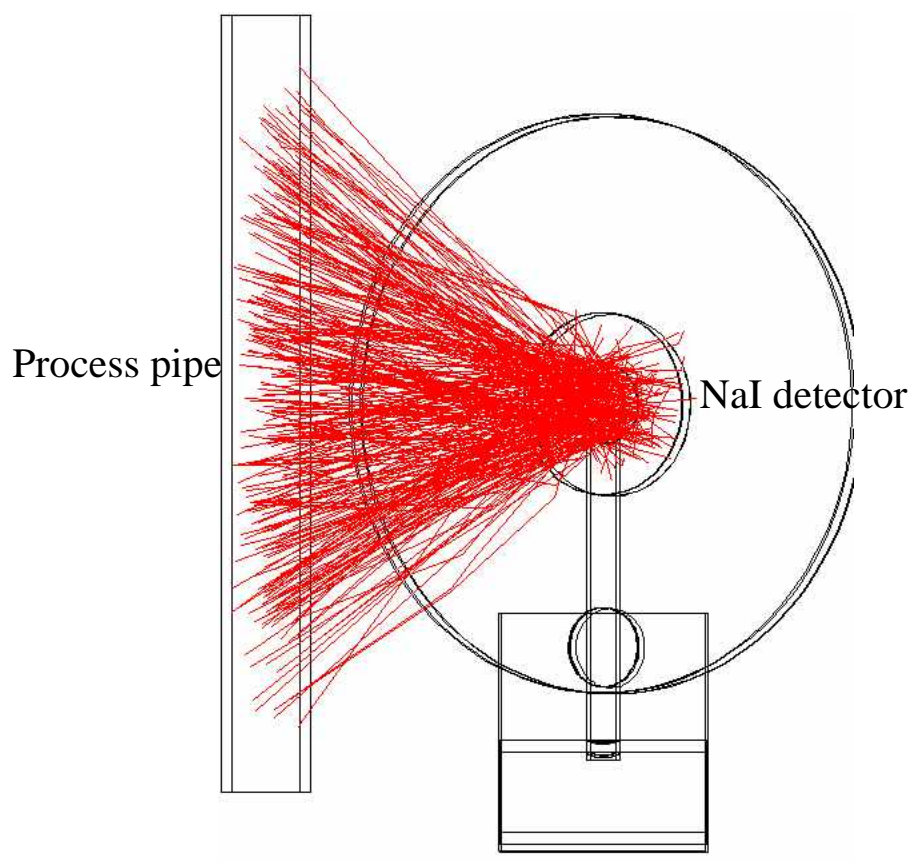

Figure 8. Line drawing showing gamma ray tracks from the process pipe to the NaI detector.

The NaI detector is used to measure the buildup or decay of the gammas from the Ba- $137 \mathrm{~m}$ daughter of Cs-137. The TRUMP card in the PC is used as a multichannel analyzer (MCA) and a region of interest (ROI) is set around the 661.7 gamma-ray peak from the Cs daughter, $\mathrm{Ba}-137 \mathrm{~m}$, and the counts in this peak summed. In order to do this, the MCA must look at all the counts from the NaI detector to determine which ones lie in the ROI. Since the MCA can only evaluate one count at a time, it is "dead" to another count while evaluating this count. Therefore, if the total number of counts is too great, the "dead time" of the MCA will be excessive, lengthening the counting time excessively. Hence both the total number of counts seen by the NaI crystal as well as the number of counts in the ROI are important.

The shielding proved to reduce the background count rate from Cs-137 in the process pipe beside the detector. The background will not add significantly to the dead time, and the counts from the process pipe will be only about $10 \%$ of the total photopeak count rate from the sample pipe. Hence, when the background is subtracted it will not affect the accuracy of the measurement.

The collimator tube from the sample pipe to the NaI detector is crucial in the response of the instrument. Too large of an aperture in the collimator will give a high count rate and excessive dead time. MCNP analyses were performed to size the collimator hole. As can be seen above, only gamma rays emitted from the sample pipe directly adjacent to the collimator hole can reach the detector. Either a collimator inner diameter of 0.375 or 0.250 inches will provide adequate collimation. The 0.250 inch collimator will provide a lower 
count rate with reduced dead time. Therefore, the 0.25 inch collimator is recommended since it will provide a greater margin of error if the Cs-137 in the stream is increased above the design of $0.1 \mathrm{Ci} /$ gallon.

\section{Detector Calibration}

NaI detectors were calibrated using Standard 68709-147 (Figure 9), purchased from Analytics Inc., Atlanta, GA. It was counted for 5 minutes with the sodium iodide detector using lead shielding with a 0.25 " lead collimator. The total counts obtained for a 5 -minute count were $478,588 \pm 1082$, providing a detector efficiency of $2.6 \%$ with an analyzer dead time of $20 \%$. Since the lead collimator hole was cone-shaped, rather than tube-shaped as will be provided in the detector shielding, the actual detector dead time with be less than $20 \%$ and will be acceptable for routine use. A standard will be placed in the shield calibration source port for detector calibration.

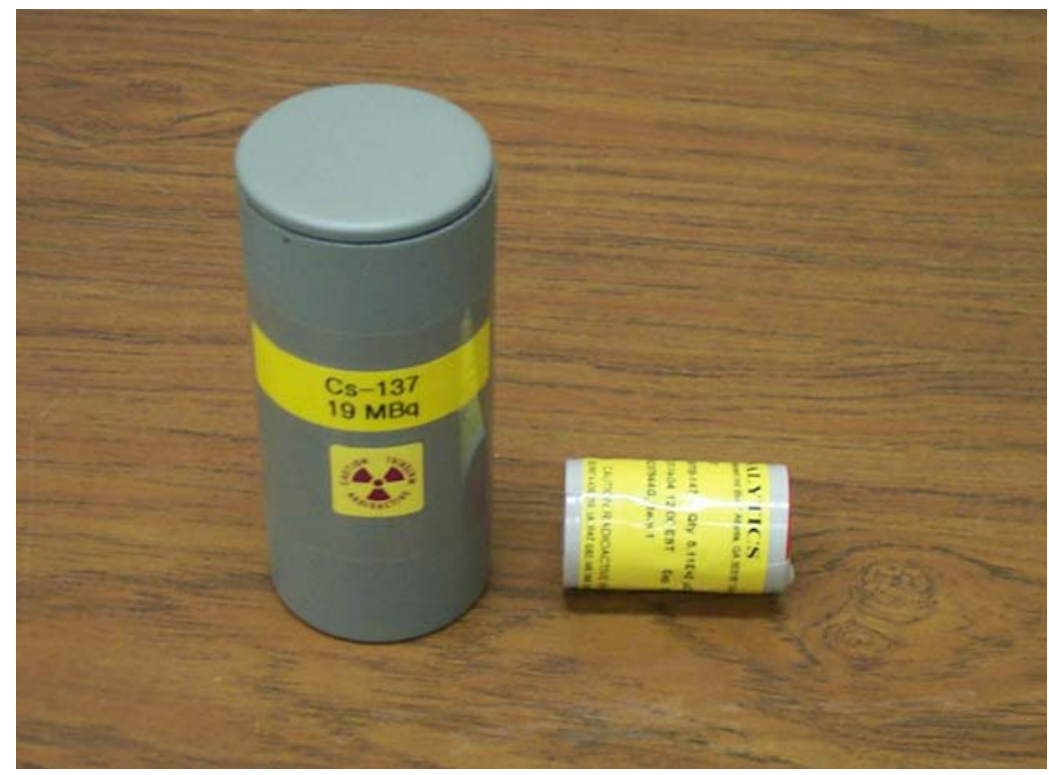

\section{Figure 9. Standard 1-in diameter $x$ 2-in long schedule 40 pipe containing $0.5 \mathrm{mCi}$ Cs-137.}

\section{System Software}

A computer program was written in Visual Basic to collect data from the process monitors and plot the data for ease of operation. A software User's Manual and software test plan were written and are given in Appendix 1 and Appendix 2.

Batch sample counting and waste transfer activities will be coordinated between the Distributed Control System (DCS) and the SCIX monitoring software by event signaling. In the batch counting mode, the DCS will notify the SCIX monitor when the sample loop for one of the NaI detectors has been filled. The SCIX monitor will perform its counting operation using the appropriate detector and notify the DCS when the operation has been completed. The SCIX monitor will provide alarm notification to the DCS upon detection of 
WSRC-RP-2004-00615

Revision 0

Page 15

such an event. In Addition, Routine Ion Exchange and Spent Resin transfer monitor operations will both be controlled via similar signals from the DCS. The SCIX monitor will also provide alarm notification to the DCS upon detection of an alarm event during either transfer operation.

\section{REEFERENCES}

1. "Task Technical Plan for Small Column Ion Exchange Gamma-Ray Monitors," WSRCRP-2003-00158, February 3, 2004. 
Appendix 1. Software User's Manual

\section{Small Column Ion Exchange Monitoring System}

\section{Software User's Manual, Rev 1}

Tim Riley

Engineered Equipment \& Systems

Savannah River National Laboratory 
WSRC-RP-2004-00615

Revision 0

Page 17

\section{TABLE OF CONTENTS}

SCIX Monitoring System Software Description ......................................................................... 18

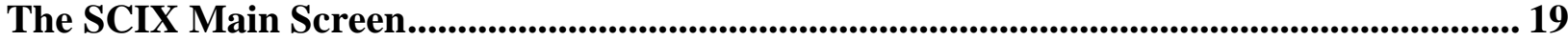

The SCIX Configuration Utility .................................................................................................. 20

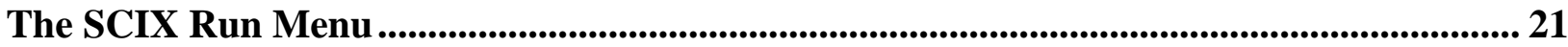

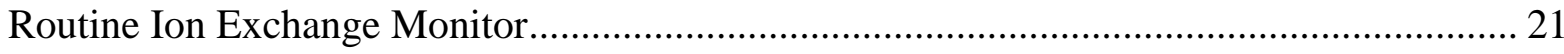

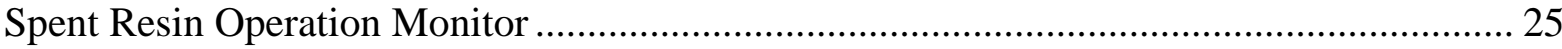

Appendix A -Log file Data Format................................................................................................ 26 


\section{SCIX MONITORING SYSTEM SOFTWARE DESCRIPTION}

The Small Column Ion Exchange (SCIX) Monitoring System is a custom Visual Basic 6.0 application program that will monitor the operation of the Small Column Ion Exchange System designed for the removal of Cs-137/Ba-137m from high-level radioactive waste streams at SRS.

The SCIX Monitoring system has two modes of operation. The first is as a Routine Ion Exchange (IX) Monitor. In this mode, the software monitors two Sodium Iodide (NaI) and one Geiger-Mueller (GM) transfer monitor and one GM breakthrough detectors. The four detectors provide a means to verify proper operation of the ion exchange system and to detect Ba-137m/Cs-137 breakthrough from the resin bed. The two GM detectors are operated in a free running mode to serve as a real time monitor for the IX operations. By default, they are set to perform continuous 60 seconds counts of the resin bed inlet and effluent piping systems. The most recent data pair is displayed on the SCIX main screen and is appended to the GM data graph.

In contrast, the $\mathrm{NaI}$ detectors are operated in batch mode with only one detector being monitored via software at any given time. The other detector serves as a backup. The user must select the appropriate NaI detector from the SCIX main screen prior to starting the monitoring operation. The operation consists of filling the batch volume from the IX effluent stream and performing 20 consecutive 60 second counts on the sampled waste volume. The 20 data points are plotted on an Ingrowth / Decay plot for determination of $\mathrm{Ba} / \mathrm{Cs}$ equilibrium. The Ba-137m activity is determined from the first reading and the Cs-137 activity from the last. The two new data points are displayed on the SCIX main screen and appended to the $\mathrm{NaI}$ data graph. Accurate operation of the NaI detector system is reliant upon up-to-date detector background and efficiency settings entered manually through the Configuration Utility. To obtain these values, the user must periodically measure the fullenergy peak background in the empty sample volume and calibrate the detector using a certified check source.

The second operating mode is the Spent Resin Operation. In this mode, two GM detectors are used as transfer monitors to observe the spent resin feed line to the grinder and the ground resin return line to the high-level waste tank. Both detectors operate in free running mode performing continuous 60 second counts for the duration of the transfer. The most recent data points are displayed on the SCIX Main screen and are appended to the Spent Resin GM Data graph. 


\section{THE SCIX MAIN SCREEN}

At startup the Small Column Ion Exchange main screen is displayed. See Figure 10. The system time is displayed in the upper right hand corner of the title bar and updated every second to indicate that the system is active.

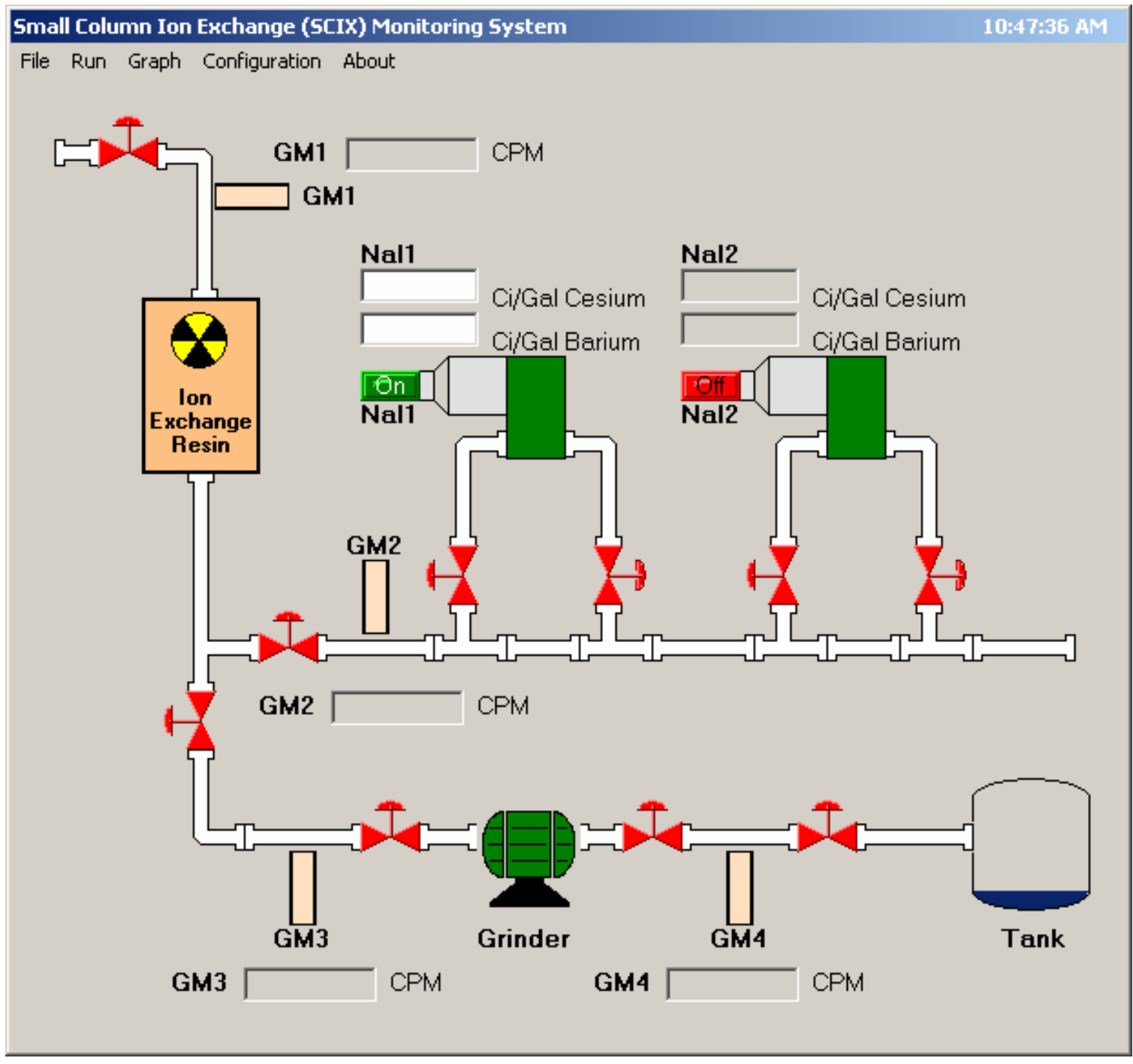

Figure 10 - SCIX Main Screen

From this screen, the User can start either the Routine Ion Exchange monitor or the Spent Resin monitor, change one of the configuration settings, or exit the system. System graphs are not visible until one of the two monitoring operations has been started. NaI detector selection is also performed from this screen by clicking on the appropriate red or green selector button on NaI1 or NaI2. Detector selection is disabled once data collection has started. 


\section{THE SCIX CONFIGURATION UTILITY}

The SCIX Configuration Utility allows the user to change system operating parameters for all six detectors (2-NaI and 4-GM Tubes.) Once changed, the new values are stored in a configuration file for future use. The user can access the configuration utility, by clicking the Configuration menu option on the SCIX main screen. Because these values are crucial to the operation of the system and its ability to produce reliable output data, a password is required to access the configuration settings. The password utility is case sensitive. The password screen is shown in Figure 11.

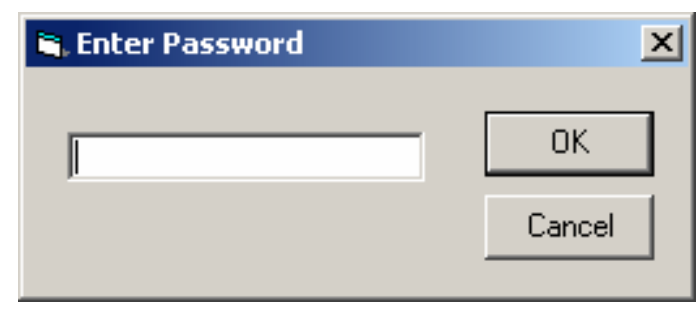

Figure 11 - Configuration Utility Password Screen

Upon entering the correct password, the user will be able to access data on the Detector Configuration screen. See Figure 12. The screen is divided into 6 sections, two identical NaI sections and four identical GM sections.

In the $\mathrm{NaI}$ setup the user can select the time interval for each count (default value is 60 seconds.) and the number of counts to be performed (default value is 20). For example, if the numbers displayed in the NaI1 section were used, the system would perform $5-1$ second counts. By default, the SCIX system will be set to perform $20-60$ second counts for each Ba-137m/Cs-137 analysis. The user can also enter values for detector efficiency and background. These values will be used in the calculation of the Ba-137m/Cs-137 concentration ( $\mathrm{mCi} / \mathrm{gal})$. The Last Update fields for each value are changed to reflect the date and time the settings were last modified. This is done programmatically and is not available to be modified by the user. The user is also able to change the system alarm setting for each detector from this screen. Range checking and data validation techniques are employed on each data entry field to force the input to an acceptable range of values. Operation of the four GM sections is identical to that described in the NaI section above.

Once all changes have been made, the User must click the Accept button to update the configuration file and use the new settings. Clicking the Close button will exit the form and return the user to the previous screen. The new settings will be discarded if the Accept button is not clicked prior to clicking the Close button. 


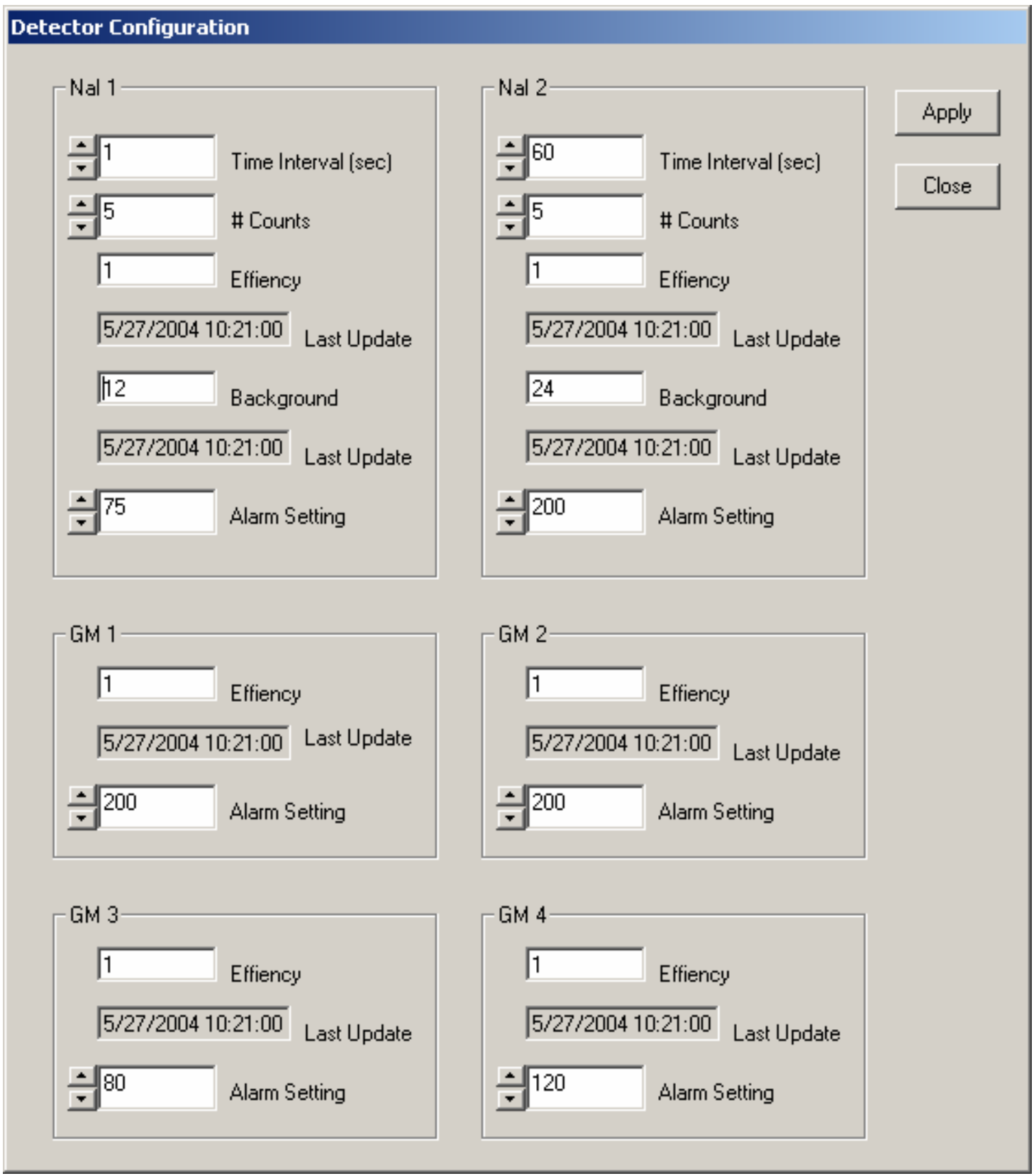

Figure 12 - Detector Configuration Screen

\section{THE SCIX RUN MENU}

\section{Routine Ion Exchange Monitor}

The SCIX Run Menu allows the user to start either of the two system monitors, the Routine Ion Exchange Monitor or the Spent Resin Operations Monitor. If the Start Routine IX option is selected two graphs will immediately be displayed. The first is the Routine Ion Exchange Data graph which contains plots for GM1 and GM2 data. See Figure 13. By default, the GM 
data is read from the NI-6602 card every 60 seconds. This value is not accessible to the user. A next-update counter in the lower left hand corner of the screen is updated once a second to show the time remaining until the next GM data pair is available.

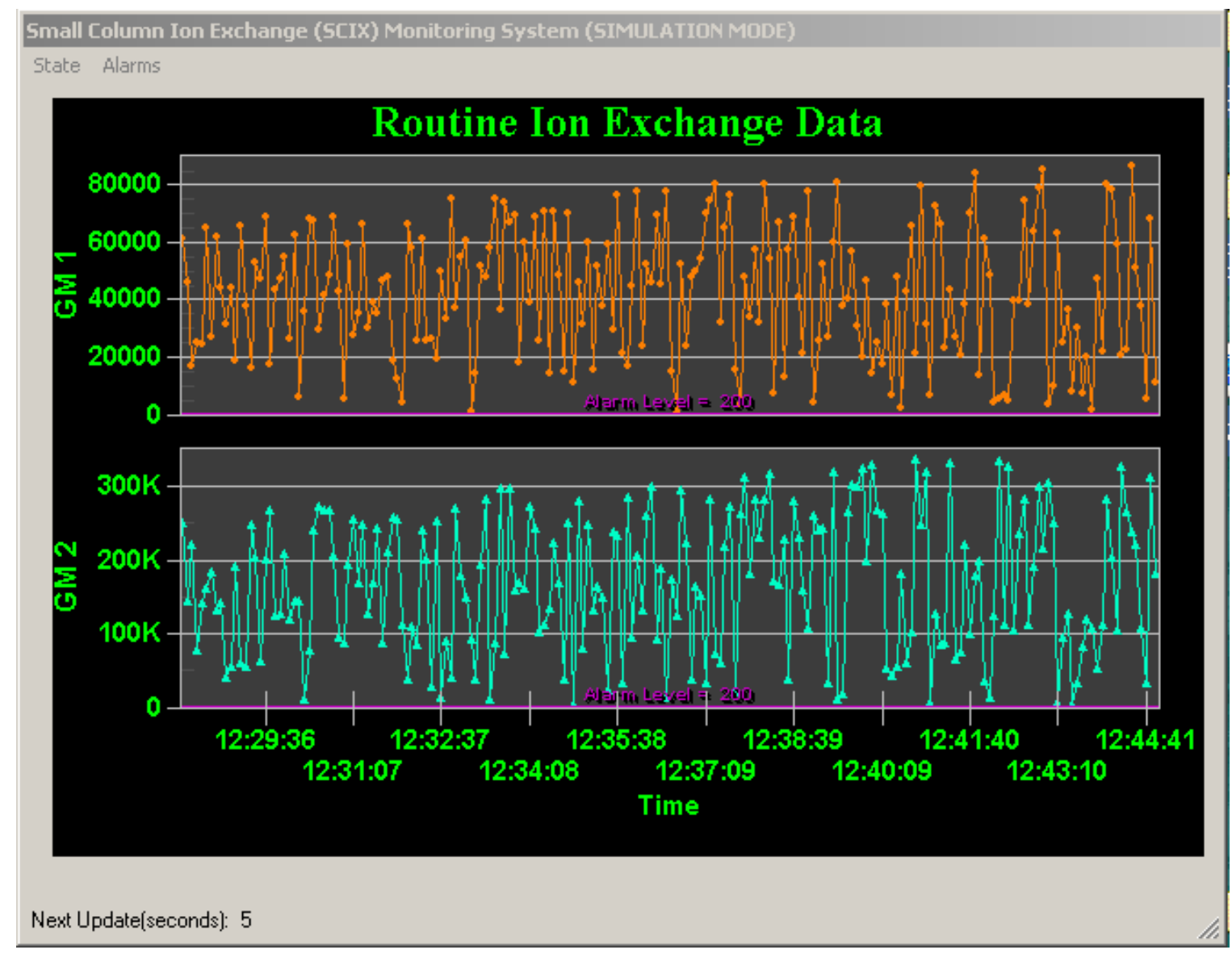

Figure 13 - Routine Ion Exchange Data - GM Graph

Figure 14 shows the second graph displayed when the Start Routine IX option is selected. The PMT data graph is simply a graph of the resin bed Ba- $137 \mathrm{~m} / \mathrm{Cs}-137$ breakthrough activity concentration in $\mathrm{Ci} / \mathrm{gal}$ as measured by the selected $\mathrm{NaI}$ detector. (Remember that the detector selection is made on the main form prior to the start of data collection.) The graph will show the 200 most recent data points collected, with only 50 displayed at any one time. The remainder of the points can be displayed via the horizontal scroll bar at the bottom of the plot. The value of any data point can be displayed in the upper left hand corner of the plot (Refer to number 41.0 in Figure 14.) by clicking on any data point. As new data is collected, the most recent data pair is added to the right side of the plot. The new data pair can be checked against the alarm value to determine if any action needs to be taken. If the Monitor is stopped, the most recent 200 data points are also saved to file and are restored to the graph at the next startup. This provides a historical trend of the resin bed breakthrough activity. 


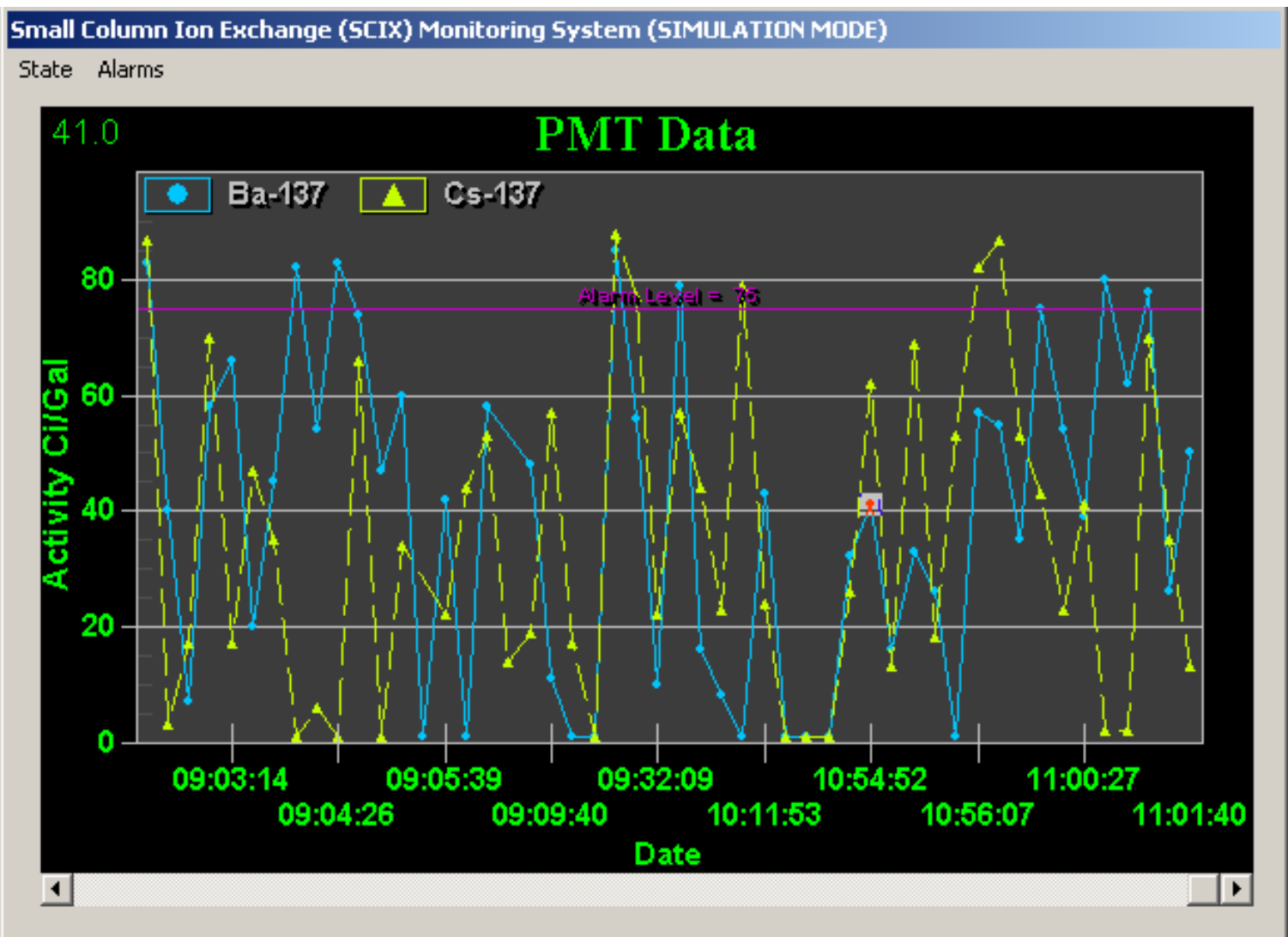

Figure 14 - PMT Data - NaI Detector Graph

Data for this plot is obtained from the Ba-137m/Cs-137 Ingrowth / Decay graph. Currently, this operation is performed manually by the operator at a frequency to be specified by procedure. The Ingrowth/Decay calculation is started by choosing the Run IG Update option from the Run menu on the SCIX Main Screen. Choosing this option will pop up the graph shown in Figure 15 and start the data collection using the selected detector through its associated Trump-PCI card. Choosing the Stop IG Update option at any time during the analysis will stop $\mathrm{NaI}$ data collection, discard the current data set, and return to the previous screen.

At the start of this operation, the program reads the $\mathrm{NaI}$ detector configuration data as set previously using the Configuration Utility. The system starts the first count by writing the live time preset (Time Interval value from the Configuration Utility) to the Trump Card to start the spectrum collection. After the time interval has elapsed, the program polls the Trump card to determine if the required live time spectrum collection has completed. If the live time interval has not completed the program waits until its completion and then reads the raw counts and other ROI statistical information from the Trump card. (These two times should be about equal, unless a significant amount of dead time is encountered.) The ROI in 
question is one that is established by the user during the initial system setup and calibration and should be the one containing the Ba-137m photo-peak.

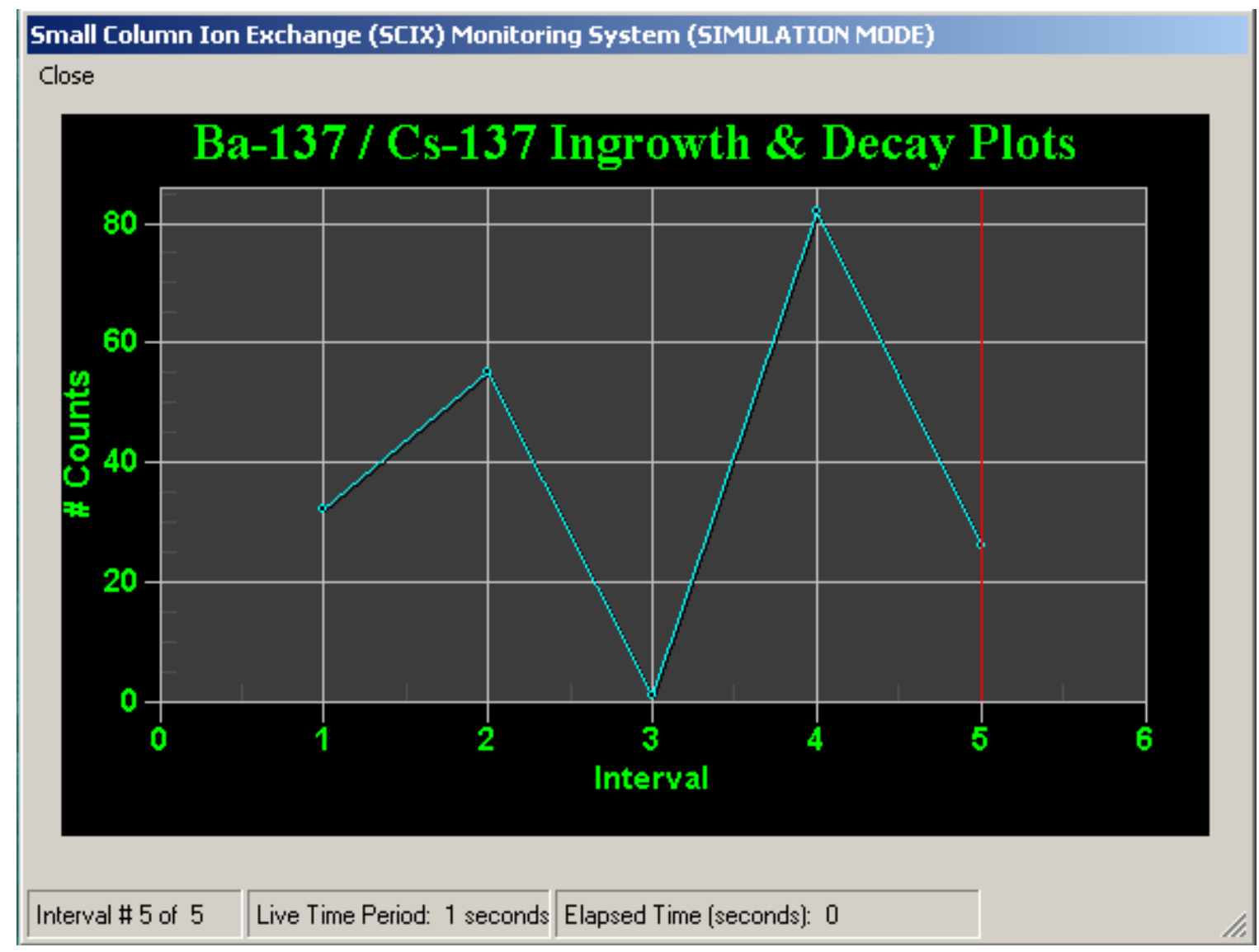

Figure 15 - Ba-137m / Cs-137 Ingrowth / Decay Graph

After the photo-peak data is collected, the concentration value is calculated by subtracting the detector background value from the raw counts in the ROI and dividing the result by the detector efficiency. This value along with other ROI and photo-peak statistical information is stored in a buffer until all the counts for an analysis have been completed. This value is plotted on the Ingrowth / Decay Graph and the entire process is repeated for the \# Counts specified in the configuration settings. The count interval, live time period, and elapsed time are displayed in the status bar area below the plot. The elapsed time value is updated each second to provide an indication of the time remaining in the current interval. The Interval\# data is also incremented for each pass.

When all 20 of the 60 second iterations have been completed the buffer containing photopeak and ROI data as well as the header information is written to a log file. The log file format is shown in Appendix A. Concentration data from the very first iteration and from the very last iteration in the analysis are written to the PMT Data graph shown in Figure 14. The first data point corresponds to $\mathrm{Ba}-137 \mathrm{~m}$ and the second to $\mathrm{Cs}-137$ breakthrough activity 
respectively. The new data points are also compared to the existing alarm setting and the appropriate sequence is initiated.

\section{Spent Resin Operation Monitor}

Choosing the Start Spent Resin Ops (SROPS) option from the Run menu will cause a single graph to be displayed. The graph contains plots for detectors GM3 and GM4, which monitor transfer lines to and from the spent resin grinder. See Figure 16. Similar to the RIX Monitor, the GM data is read from the NI-6602 card every 60 seconds. This value is not accessible to the user. A next-update counter in the lower left hand corner of the screen is updated once a second to show the time remaining until the next GM3 \& GM4 data pair is available.

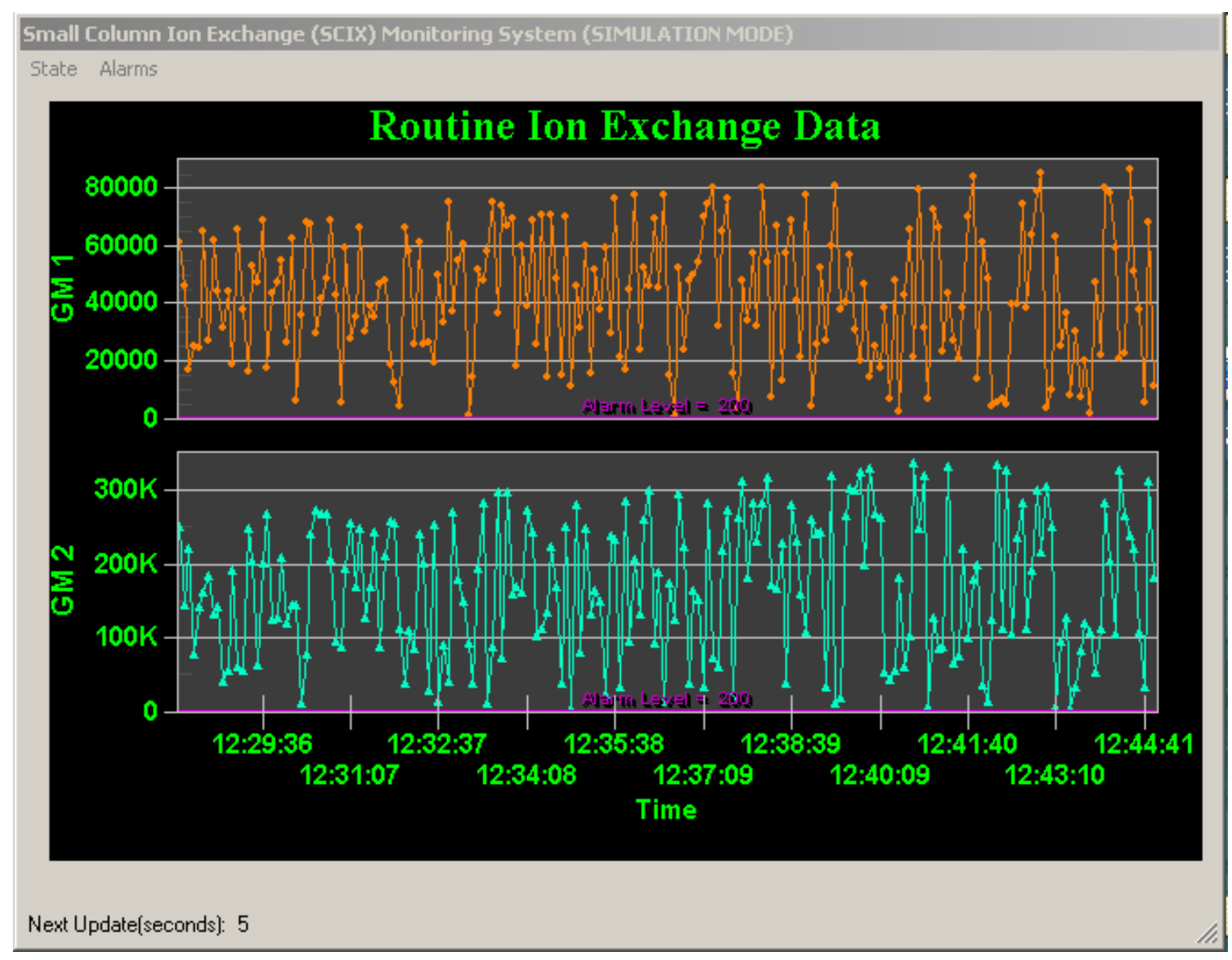

Figure 16 - Spent Resin Operation - GM Graph 


\section{APPENDIX A -LOG FILE DATA FORMAT}

The format for the Log file data is shown in Table A-1 below. At the start of each analysis, the Header Data is obtained from the Configuration Utility and stored in the buffer area. When the first iteration has completed, the Iteration Data is appended to the end of the buffer containing the Header Data. Data from ensuing iterations is appended to the end of the buffer until the required number of iterations in the analysis has been completed. At this point, the "End of Data" marker is added and the entire buffer contents written to the log file. Data from a complete analysis is shown in Table A-2.

\section{Table A-1 - Log File Data Format}

\author{
HEADER INFO \\ Date \& Time: \\ Detector Background: \\ Background Date: \\ Detector Efficiency: \\ Efficiency Date: \\ Detector Selected:
}

ITERATION DATA

Iteration\#:

Concentration:

Live Time:

True Time:

ROI Start:

ROI Channels:

Peak Channel:
Date and time that analysis was started.

Background data as set in the Configuration Utility.

The last time the Detector Background setting was updated.

Efficiency data as set in the Configuration Utility.

The last time the Detector Efficiency setting was updated.

The detector used in the analysis \#1-NaI1, \#2-NaI2

The cycle number for the current analysis.

The Ba-137/Cs-137 concentration calculated using the detector background and detector efficiency settings listed in the header info.

The live time setting for each iteration. (The number of $20 \mathrm{msec}$ intervals)

The true time elapsed for the iteration, as read from the Trump Card. (The number of 20 msec intervals)

The starting channel number of the ROI.

The number of channels in the ROI.

The channel number of the photo-peak centroid.

(Repeat Iteration Data for each loop)

End of Data Marker:

(END OF DATA:) A flag to signal the end of data for the current analysis. 
WSRC-RP-2004-00615

Revision 0

Page 27

\section{Table A-2 - Typical Analysis Data Example}

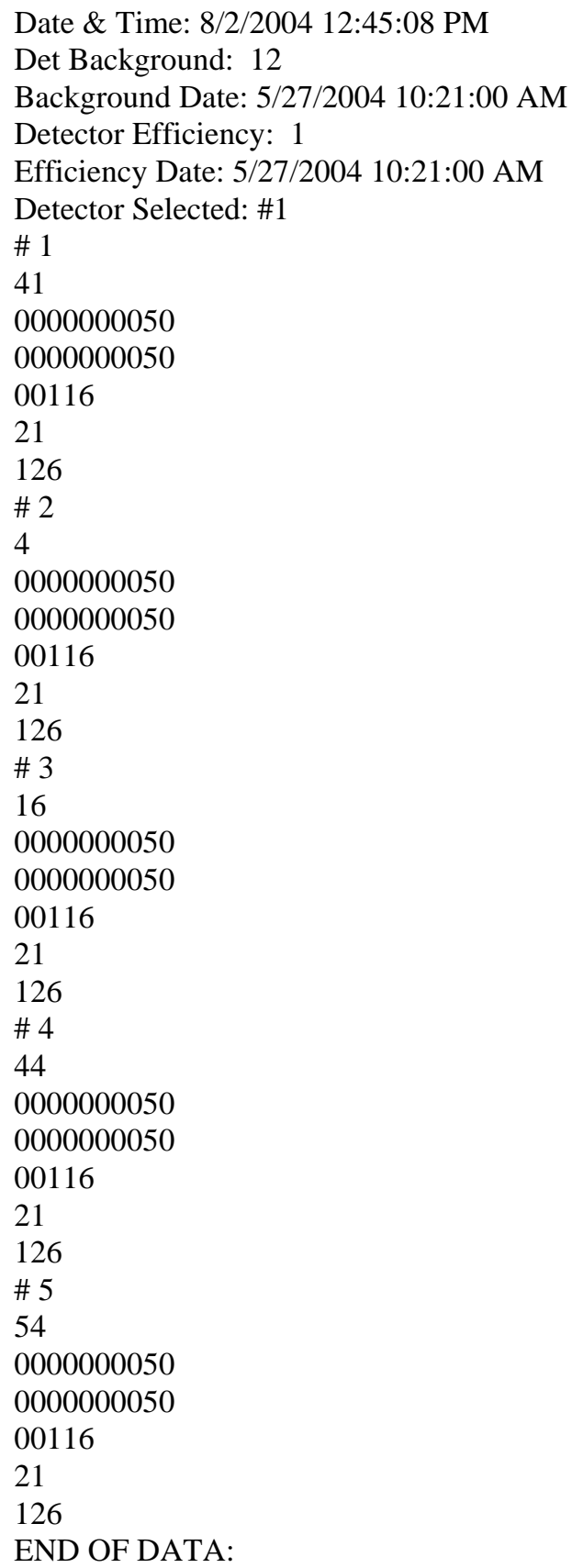


Appendix 2-Software Test Plan

\section{Small Column Ion Exchange Monitoring System}

\section{Software Test Plan, Rev 0}

Tim Riley

Engineered Equipment \& Systems

Savannah River National Laboratory 


\section{Main Form}

1. At start up verify that the clock is functional in the URH Corner of the SCIX Main form.

2. Verify that NaI probe selection works properly by toggling between ON/OFF states when either selection button is clicked.

\section{Configuration Menu}

1. Verify that the password box takes the correct password and rejects the others.

2. Verify that all fields in the Configuration Utility are populated when the Config form is displayed.

3. Verify that range checking works on all input fields and that non numeric entries are rejected. Test for all 6 detectors.

4. Verify that date is changed to the current date and time when new values are entered for either detector background or detector efficiency and Apply button is clicked. Test for all six detectors.

5. Verify that all changes are rejected on the Config form if the Apply Button is not clicked. To verify the user must click the Close button and then reopen the Config Utility and check the contents of the field that was changed. Test for all fields.

\section{Graph Menu}

1. Verify that all menu options are grayed out.

\section{Run Menu}

1. Click the Run menu option. Verify that the only two options that are available are Start Routine IX and Start Spent Resin Ops. All other options should be grayed out.

2. Click the Start Routine IX option and verify that 2 graphs are shown. PMT data graph and the GM1\&2 graphs.

3. Under the Graph menu option verify that the Show NaI Graph and Show GM Graph menu options are available and the all others are grayed out

4. Verify that the GM graph is working by observing the Next Update filed at the LLH corner of the screen. The field should countdown to zero and then new data values should appear on both the GM1 and GM2 plots and on the SCIX main screen.

5. Verify that the PMT Data graph is not receiving updates, but that the graph displays the last 200 points obtained. (May not contain all 200 points if the system has not been operated that long.)

6. Click the Run ID Update menu option.

7. Verify that the File Dialog Box appears prompting the location to save the log file.

8. Verify that the Stop ID Update and the Stop Routine IX menu options are now available. All others should be grayed out.

9. Verify that the Ingrowth / Decay (ID) graph is shown and is operating. The elapsed time field at the bottom of the form should update once per second.

10. Click the Stop ID Update menu option and verify that the ID graph disappears and that the data collected is not written to the PMT Data graph.

11. Click the Run ID Update menu option and again verify that the ID graph is displayed and is operating. 
12. At the end of the time interval (60 seconds) a new data value pair should be displayed on the plot and the next count interval started.

13. When all intervals have completed, the data should be plotted on the PMT Data form. Verify that the first value from the ID Graph is plotted as the Ba-137 entry and the last is plotted as the Cs-137 entry on the PMT Data graph. These values should also appear on the SCIX main screen

14. Click the close button on the top of the ID graph and verify that the graph disappears.

15. Verify that the Start ID Update and Stop Routine IX menu options are available; all others should be grayed out.

16. Again click the Run ID Update menu option and verify that the ID graph is displayed and is operating.

17. Click the Stop ID Update menu option to close the ID graph.

18. Verify that the alarm levels displayed on the GM1 \& 2 graph and on the PMT Data graph are consistent with the settings in the Configuration Utility.

19. Verify that changes made the alarm settings in the Configuration Utility appear on both graphs.

20. Click the Stop Routine IX option and verify that the 2 graphs disappear and that the menu options are back to that specified in step 1. Also verify that all menu options on the Graph menu are grayed out.

21. Click the Start Spent Resin Ops option and verify that the GM3\&4 graph is shown.

22. Under the Graph menu option verify that the Show Spent Resin Graph menu option is available and the all others are grayed out.

23. Verify that the GM graph is working by observing the Next Update filed at the LLH corner of the screen. The field should countdown to zero and then new data values should appear on both the GM3 and GM4 plots and on the SCIX main screen.

24. Verify that the alarm levels displayed on the GM3 \& 4 graph are consistent with the settings in the Configuration Utility.

25. Verify that changes made the alarm settings in the Configuration Utility appear on both graphs.

26. Click the Stop Spent Resin Ops option and verify that the GM3 \& 4 graph disappears and that the menu options are back to that specified in step 1. Also verify that all menu options on the Graph menu are grayed out.

\section{File Menu}

1. Click the Exit menu option under the File Menu and verify that the application closes.

\section{Data File Verification}

1. Open log file and verify that header data is correct. Also verify that data is recorded for all iterations of the ID Update run. 